\title{
Coleções subdurais crônicas não infecciosas: Aspectos epidemiológicos e resultados em 581 pacientes consecutivos submetidos a tratamento cirúrgico
}

\author{
Non-infectious chronic subdural collections: epidemiological aspects and results in 581 \\ consecutive operated patients
}

Tamara Melissa Zavadzki ${ }^{1}$, Guilherme Henrique Ferreira da Costa ${ }^{2}$, Leonardo Henrique da Silva Rodrigues ${ }^{2}$, Paulo Adolfo Wessel Xander ${ }^{2}$, José Carlos Esteves Veiga ${ }^{3}$, Guilherme Brasileiro de Aguiar ${ }^{4}$

\section{Resumo}

Introdução: Coleções subdurais crônicas, representadas na sua maioria pelos hematomas (HSDCr) e higromas (HSD), são afecções frequentes na prática neurocirúrgica. Sua apresentação clínica, assim como as técnicas cirúrgicas empregadas para o seu tratamento, já são bem documentadas na literatura. Objetivo: O presente estudo tem como objetivo traçar o perfil epidemiológico dos pacientes diagnosticados com coleções subdurais crônicas, representadas por $\mathrm{HSDCr}$ e HSD, submetidos a tratamento neurocirúrgico no nosso serviço, no período de 2000 a 2014. Métodos: Foram analisados os relatos cirúrgicos entre o período de 2000 a 2014, de pacientes diagnosticados com coleções subdurais crônicas, compreendendo hematomas e higromas. Avaliaram-se características demográficas, tipo de tratamento empregado, ocorrência de recidivas, lateralidade, localização

1. Médica Residente da Irmandade da Santa Casa de Misericórdia de São Paulo - $1^{\circ}$ Ano da Disciplina de Neurologia

2. Médicos Residentes da Irmandade da Santa Casa de Misericórdia de São Paulo - do $2^{\circ}$ ano da Disciplina de Neurocirurgia

3. Chefe da Disciplina de Neurocirurgia da Irmandade da Santa Casa de Misericórdia de São Paulo e Professor Titular da Faculdade de Ciência Médicas da Santa Casa de São Paulo-Departamento de Cirurgia

4. Professor Instrutor da Faculdade de Ciência Médicas da Santa Casa de São Paulo - Departamento de Cirurgia. Médico Assistente da Irmandade da Santa Casa de São Paulo-Disciplina de Neurocirurgia

Trabalho realizado: Irmandade da Santa Casa de Misericórdia de São Paulo - Departamento de Cirurgia, Disciplina de Neurocirurgia

Endereço para correspondência: Guilherme Henrique Ferreira da Costa. Avenida São João, número 1459, apto 43, Santa Cecília, 01211-100 - São Paulo-SP. E-mail: guilhermecosta@outlook.com Declaração de Conflito de Interesses: Os autores declaram não haver conflito de interesse.

Certificado de Apresentação para Apreciação Ética: Parecer número 1.051.510 de 08/05/2015. e manutenção de dreno no pós-operatório. Resultados: Neste estudo, foram selecionados 581 pacientes, dentre os quais 500 (86,05\%) apresentaram HSDCr e 81 (13,9\%) HSD. Eram do sexo masculino $74,18 \%$. Em relação aos HSDCr, a média de idade foi $61,6 \pm 21$ anos. A trepanação foi o tipo de intervenção mais utilizada (95\%), seguido de craniotomia (4,7\%). Houve recidiva em 29 casos. No que diz respeito aos $H S D$, a média de idade foi $45,7 \pm 27,2$. A trepanação foi realizada em 91,5\% dos casos, seguida pela craniotomia $(3,16 \%)$, derivação subduro-peritoneal $(3,1 \%)$ e derivação subdural externa (2,1\%). Houve 15 casos recidivados. Conclusão: Os HSDCr representam a maioria das coleções subdurais submetidas a tratamento cirúrgico. Os idosos foram o grupo mais acometido. O tratamento cirúrgico de escolha foi a trepanação.

Descritores: Hematoma subdural crônico, Hematoma subdural, Derrame subdural

\footnotetext{
Abstract

Introduction: Chronic subdural collections, represented mostly by hematomas (HSDCr) and hygromas (HSD), are frequent conditions in neurosurgical practice. Its clinical presentation, as well as the surgical techniques used for its treatment, are already well documented in the literature. Objective: This study aims to trace the epidemiological profile of patients diagnosed with chronic subdural collections, represented by HSDCr and HSD, submitted to neurosurgical treatment at our Division, from 2000 to 2014. Methods: Surgical reports between 2000 and 2014 were analyzed, patients diagnosed with chronic subdural collections, including hematomas and hygromas, were selected. Demographic characteristics, type of treatment used, occurrence of relapses, laterality, location and drainage maintenance were evaluated in the postoperative period. Results: In this study, 581 patients were selected, of which $500(86.05 \%)$ presented HSDCr and 81 (13.9\%) HSD. They were male gender in $74.18 \%$. Regarding HSDCr, the mean age was $61.6 \pm 21$
} 
Zavadzki TM, Costa GHF, Rodrigues LHS, Xander PAW, Veiga JCE, Aguiar GB. Coleções subdurais crônicas não infecciosas: Aspectos epidemiológicos e resultados em 581 pacientes consecutivos submetidos a tratamento cirúrgico. Arq Med Hosp Fac Cienc Med Santa Casa São Paulo. 2018;63(2):85-9.

years. Trepanation was the most used type of intervention (95\%), followed by craniotomy (4.7\%). There were recurrences in 29 cases. With regard to $H S D$, the mean age was $45.7 \pm 27.2$. Trepanation was performed in $91.5 \%$ of cases, followed by craniotomy (3.16\%), subdural-peritoneal shunt (3.1\%) and external subdural shunt (2.1\%). There were 15 recurrences. Conclusion: HSDCr represent the majority of subdural collections submitted to surgical treatment. The elderly were the most affected group. The surgical treatment of choice was trepanation.

Keywords: Hematoma, subdural, chornic; Hematoma, subdural; Subdural effusion

\section{Introdução}

As coleções subdurais crônicas, representadas na sua maioria pelos hematomas (HSDCr) e higromas (HSD), são afecções frequentes na prática neurocirúrgica. Os HSDCr constituem apresentação comum de hemorragia intracraniana. Estão associados comumente a traumatismos cranioencefálicos prévios, geralmente de leve intensidade, com taxas de morbidade e mortalidade relativamente altas ${ }^{(1-2)}$, ocorrendo principalmente em idosos. Outra lesão que se assemelha ao HSDCr é o Higroma Subdural, sendo que a distinção entre ambos permanece controversa. Se o conteúdo fluido é claro e xantocrômico ou com pequena quantidade de sangue denomina-se higroma subdural, enquanto que o hematoma subdural se caracteriza por ter um fluido escuro extremamente sanguinolento, comparado a óleo de motor. $\mathrm{Na}$ investigação diagnóstica por tomografia computadorizada, o coeficiente de atenuação (densidade) do higroma subdural traumático apresenta aspecto semelhante ao do HSDCr hipodenso, o que dificulta a diferenciação ${ }^{(3)}$.

Haja vista o progressivo envelhecimento da população mundial observa-se, atualmente, aumento na incidência das coleções subdurais crônicas. Isso promove maior impacto na qualidade de vida do indivíduo, refletindo em custos tanto sociais quanto econômicos para as populações envolvidas ${ }^{(2)}$. O processo de atrofia cerebral constitui fator de risco significativo associado principalmente aos idosos e etilistas crônicos. Além disso, traumatismos cranioencefálicos prévios, mesmo de leve intensidade, como quedas da própria altura, estão associados a aproximadamente $60 \%$ dos pacientes ${ }^{(4-6)}$.

A apresentação clínica, assim como as técnicas cirúrgicas empregadas para o tratamento, já são bem estabelecidas. No entanto, com o envelhecimento da população, as características demográficas da população acometida vêm sofrendo variações. Há poucos estudos que descrevam com precisão as características epidemiológicas dos pacientes acometidos por estas condições em nosso país. Deste modo, o presente estudo tem como objetivo caracterizar o perfil epidemiológico dos pacientes diagnosticados com coleções subdurais crônicas não infecciosas submetidas a tratamento neurocirúrgico em nosso serviço, no período de 2000 a 2014.

\section{Métodos}

Trata-se de um estudo transversal retrospectivo realizado através da análise de prontuários referentes a pacientes diagnosticados com coleções subdurais crônicas de etiologia não infecciosa submetidos a tratamento neurocirúrgico em um único centro de referência localizado na região metropolitana de São Paulo-SP, no período de 2000 a 2014.

Foram excluídos todos os casos de coleções infecciosas, subdurais agudas e subagudas.

Foi iniciada coleta de dados após aprovação do projeto junto ao comitê de ética e pesquisa de nossa instituição sob número 1.051.510 de 08/05/2015. Dado seguimento ao projeto, avaliamos os relatos cirúrgicos presentes nos prontuários de todos os pacientes diagnosticados com coleções subdurais crônicas não infecciosas, compreendendo hematomas e higromas, no período de 2000 a 2014. O método de imagem utilizado foi a Tomografia Computadorizada de Crânio.

Os dados obtidos foram organizados de acordo com as características demográficas, tipo de tratamento cirúrgico empregado (trepanação, craniotomia ou derivações temporárias), taxas de recidivas, lateralidade e localização.

Para cálculo da mediana de idade dos pacientes, foi utilizada ferramenta de cálculo de mediana crescente no programa Microsoft office Excel.

\section{Resultados}

De acordo com a Tabela 1, a amostra consistiu em 581 pacientes consecutivos, 431 eram do sexo masculino $(74,18 \%)$ e 150 do sexo feminino $(25,82 \%)$. Dos casos avaliados, $500(86,05 \%)$ apresentaram HSDCr e 81 pacientes $(13,95 \%)$ HSD. No que condiz ao tratamento cirúrgico, a trepanação foi a técnica mais utilizada, realizada 591 vezes $(94,56 \%)$

Analisando-se individualmente os casos de HSDCr, observamos que nos pacientes avaliados, sem levar em consideração o gênero, a idade apresentou os limites de variação entre o primeiro dia de vida e os 97 anos de idade, sendo a mediana obtida de 66 anos.

Dos pacientes acima de 65 anos, 177 eram homens $(67,56 \%)$ e 85 mulheres (32,44\%), representando $52,40 \%$, vide Tabela 2 .

Foram, ao todo, realizadas 529 cirurgias para tratamento do HSDCr, sendo contabilizadas as de trata- 


\section{Tabela 1}

Distribuição das coleções subdurais quanto ao gênero, tipos e tratamento cirúrgico realizado.

\begin{tabular}{lcc}
\multicolumn{1}{c}{ Variável } & $N$ & $\%$ \\
Gênero & & \\
$\quad$ Masculino & 431 & 74,18 \\
$\quad$ Feminino & 150 & 25,82 \\
Coleção Subdural Crônica & & \\
$\quad$ HSDCr* & 500 & 86,05 \\
HSD** & 81 & 13,95 \\
Cirurgias Totais Realizadas & & \\
$\quad$ HSDCr* & 530 & 84,80 \\
$\quad$ HSD** & 95 & 15,20 \\
Tipo de Cirurgia & & \\
Trepanação & 591 & 94,56 \\
$\quad$ Craniotomia & 28 & 4,48 \\
$\quad$ Derivação Subduro-peritoneal & 4 & 0,64 \\
$\quad$ Derivação Subdural Externa & 2 & 0,32 \\
\hline
\end{tabular}

HSDCr*- hematoma subdural crônico. HSD**- Higroma. Fonte: Arquivos da Disciplina de Neurocirurgia da ISCMSP - Irmandade da Santa Casa de Misericórdia de São Paulo.

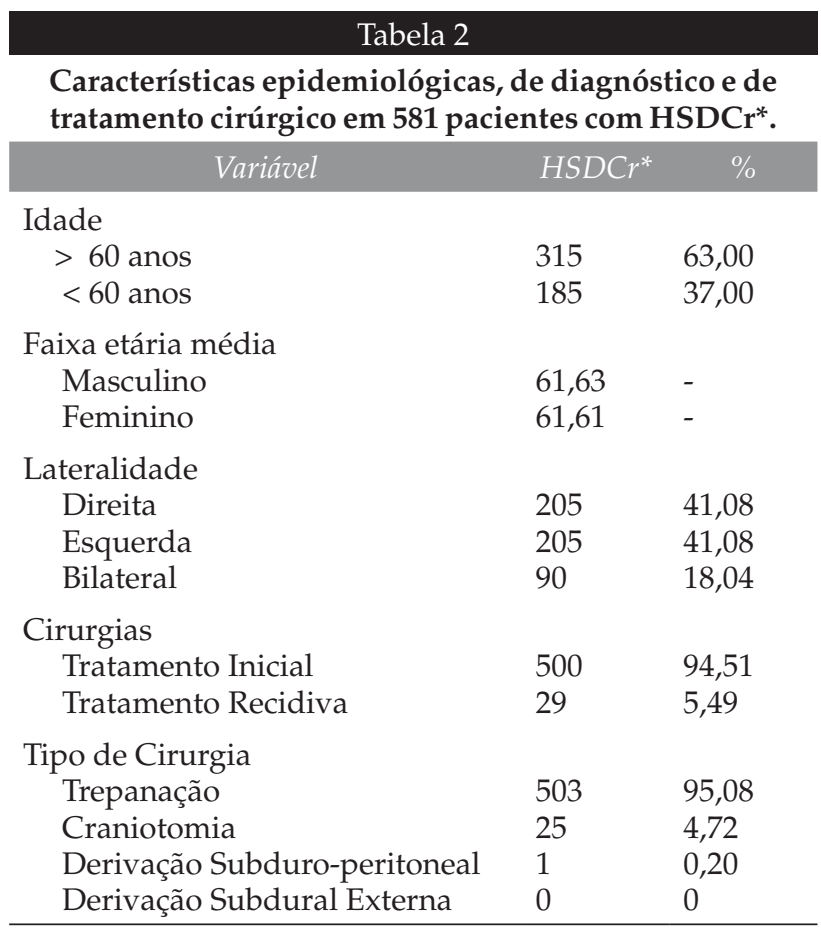

HSDCr*- hematoma subdural crônico. HSD**- Higroma. Fonte: Arquivos da Disciplina de Neurocirurgia da ISCMSP - Irmandade da Santa Casa de Misericórdia de São Paulo.

mento inicial e recidivas. A princípio foram realizadas 478 trepanações e 22 craniotomias para tratamento inicial dos pacientes com HSDCr. O tratamento mais utilizado no serviço foi a trepanação, realizada 503 vezes, contabilizando o tratamento inicial e recidiva, representando $95,08 \%$ dos casos, conforme mostra a Tabela 3.
Tabela 3

Tipo de cirurgia realizada em pacientes com $\mathrm{HSDCr}^{*}$ recidivado.

\begin{tabular}{lll}
\multicolumn{1}{c}{ Variável } & $H^{2} \mathrm{Cr}^{*}$ & \multicolumn{1}{c}{$\%$} \\
Sexo & \multicolumn{2}{c}{} \\
Masculino & 19 & 70,37 \\
Feminino & 8 & 29,63 \\
Tipo de Cirurgia & & \\
Trepanação & 25 & 86,20 \\
Craniotomia & 3 & 10,34 \\
Derivação Subduro-peritoneal & 1 & 3,44 \\
Derivação Subdural Externa & 0 & - \\
\hline
\end{tabular}

HSDCr*- hematoma subdural crônico.

Fonte: Arquivos da Disciplina de Neurocirurgia da ISCMSP - Irmandade da Santa Casa de Misericórdia de São Paulo.

Vinte e sete pacientes com diagnóstico de HSDCr necessitaram de outra intervenção cirúrgica para o tratamento das recidivas. Nestes casos, a trepanação também foi a técnica cirúrgica mais empregada, realizada em $86,20 \%$ dos casos, conforme mostrado na Tabela 3. Foram realizadas o total de 29 cirurgias nos pacientes com recidivas de HSDCr, visto que alguns pacientes foram operados mais de uma vez.

Neste estudo, devido as bases fisiopatológicas serem distintas, uma análise exclusiva dos Higromas subdurais evidenciou limites de variação de idade entre 3 meses e 89 anos, sendo a média de idade, quando não levado em consideração o sexo, de 45,72 anos. Diferentemente dos casos de HSDCr, como mostra a Tabela 4, a prevalência encontrada em HSD foi de pacientes abaixo dos 60 anos de idade, observada em $49 \operatorname{casos}(39,51 \%)$.

Ainda na Tabela 4, no que diz respeito à lateralidade encontrada nos casos de HSD, o lado esquerdo foi mais acometido em relação ao direito. Entretanto, diferentemente do encontrado em relação aos HSDCr, a maioria dos casos de HSD ocorreu bilateralmente.

Foram realizadas ao todo 95 cirurgias para o tratamento de HSD, contabilizando as cirurgias de tratamento inicial e de recidivas. O tipo de procedimento cirúrgico mais realizado, assim como nos casos de HSDCr, foi a trepanação, presente 87 vezes, representando $91,58 \%$ dos casos.

Como pode ser visto na Tabela 5 , a reoperação devido à recidiva de HSD, ocorreu em 12 casos. Nestes pacientes, foram realizadas 15 cirurgias, contabilizando os casos que necessitaram de mais de um procedimento cirúrgico para o tratamento da recidiva. Em casos recidivados a trepanação também foi a cirurgia mais realizada.

\section{Discussão}

A prevalência das coleções subdurais crônicas en- 
Tabela 4

Características epidemiológicas, de diagnósticos e de tratamento cirúrgico em 81 pacientes com diagnóstico de HSD*.

\begin{tabular}{lll}
\multicolumn{1}{c}{ Variável } & HSD** & $\%$ \\
Idade & & \\
$\quad>60$ anos & 32 & 39,50 \\
$<60$ anos & 49 & 60,49 \\
Média de Idade & & \\
$\quad$ Masculino & 46,84 & - \\
$\quad$ Feminino & 42,08 & - \\
Lateralidade & & \\
$\quad$ Direito & 26 & 41,08 \\
$\quad$ Esquerdo & 28 & 41,08 \\
$\quad$ Bilateral & 41 & 18,04 \\
Cirurgias & & \\
$\quad$ Tratamento Inicial & 80 & 84,21 \\
$\quad$ Recidiva & 15 & 15,78 \\
Tipo de Cirurgia & & \\
$\quad$ Trepanação & 87 & 91,58 \\
$\quad$ Craniotomia & 3 & 3,16 \\
$\quad$ Derivação Subduro-peritoneal & 3 & 3,16 \\
$\quad$ Derivação Subdural Externa & 2 & 2,11 \\
\hline
\end{tabular}

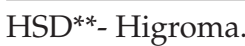

Fonte: Arquivos da Disciplina de Neurocirurgia da ISCMSP - Irmandade da Santa Casa de Misericórdia de São Paulo.

Tabela 5

Tipo de procedimento cirúrgico realizado em pacientes com HSD** recidivado.

\begin{tabular}{lcc}
\multicolumn{1}{c}{ Variável } & $H_{S D}^{* *}$ & $\%$ \\
Sexo & & \\
Masculino & 10 & 83,33 \\
Feminino & 2 & 16,67 \\
\hline Tipo de Cirurgia & & \\
Trepanação & 10 & 66,66 \\
Craniotomia & 1 & 6,66 \\
Derivação Subduro-peritoneal & 3 & 20,00 \\
Derivação Subdural Externa & 1 & 6,66 \\
\hline
\end{tabular}

HSD $^{* *}$ - Higroma.

Fonte: Arquivos da Disciplina de Neurocirurgia da ISCMSP - Irmandade da Santa Casa de Misericórdia de São Paulo.

contradas em nosso serviço foi $86 \%$, este dado é semelhante ao encontrado em um estudo recém publicado, realizado por Yang e Huang $(2017)^{(7)}$, pesquisadores da Johns Hopkins University School of Medicine, após revisão sistemática mostra que a incidência de HSDCr está aumentando, levantando a hipótese de que esse fato seja atribuído ao envelhecimento da população e crescente prevalência de uso de medicações antiagregante plaquetários e a anticoagulantes na população geral ${ }^{(7)}$.

Avaliando-se o resultado encontrado em relação à lateralidade, do HSDCr notamos prevalência semelhante, uma vez que tivemos aproximadamente $41 \%$ dos casos para cada lado, ou seja $82 \%$ dos casos são unilaterais, sendo a parcela restante, $18 \%$, referente aos casos onde foram acometidos ambos os lados. Hsieh et al, 2016 $6^{(8)}$ em estudo publicado recentemente encontraram prevalência de $30,7 \%$ para os HSDCr bilaterais, número acima dos $18 \%$ encontrados em nosso trabalho, entretanto ainda de acordo com com o fato de que a maioria dos casos encontrados é de localização unilateral $^{(8)}$. Visto este estudo ser retrospectivo, não temos dados ao que diz respeito às causas dos HDSCr.

O procedimento cirúrgico mais realizado nos casos de HSDCr estudados foi a trepanação, correspondendo a cerca de $95 \%$ dos casos. A craniotomia foi utilizada em pouco mais de $4 \%$ dos casos, e a derivação subduroperitoneal, realizada em apenas 1 paciente, representando menos de $1 \%$ do total de casos. De modo semelhante o artigo publicado ano passado por Abecassis e Kim (2017) ${ }^{(9)}$ do Harborrview Medical Center - University of Washington, apresentou dados que vão a favor do que é encontrado neste trabalho. Neste estudo, recomendam a trepanação como a estratégia inicial para o tratamento cirúrgico do HSDCr, sendo a craniotomia considerada em casos de recorrência ou na presença de coágulos com evidências de membranas espessas. Entretanto, a comparação direta entre as diferentes técnicas cirúrgicas necessita melhores estudos ${ }^{(9)}$.

Importante destacar que do total de 530 procedimentos realizados, 28 foram em pacientes que necessitaram de reoperação para tratamento de recidiva, porém até neste grupo a trepanação foi o procedimento mais realizado, representado por pouco mais de $86 \%$ dos casos. Gernsback et al, 2016 ${ }^{(10)}$ em artigo publicado na revista World Neurosurgery, encontraram taxa de recidiva em 6,1\% dos casos. Aspecto interessante observado pelos referidos autores foi o fato dos pacientes com doença hepática apresentarem maior probabilidade de recorrência. Entretanto, outras comorbidades não tiveram aumento significativo. A taxa de recorrência é semelhante a encontrada em nosso trabalho, onde ocorreram $5,2 \%$ de recidivas ${ }^{(10)}$.

O HSD é uma coleção semelhante ao líquor: apresentando conteúdo líquido claro, xantocrômico ou levemente hemorrágico. O higroma tem pressão oncótica mais baixa que a do sangue, e menor quantidade de eritrócitos, leucócitos, concentração total de proteínas, albumina e imunoglobulina G que o plasma, porém maior que o liquor ${ }^{(11)}$. Sua formação geralmente ocorre após traumatismos crânioencefálicos (TCE). No entanto, também pode ocorrer após craniotomia para tratamento de outras afecções neurocirúrgicas. Tende a diminuir sem nenhuma intervenção, mas pode não reabsorver espontaneamente e até aumentar, tornando-se um processo expansivo que leva a compressão cerebral, necessitando de intervenção cirúrgica. O quadro clínico do higroma geralmente é 
inespecífico, com distúrbios da consciência sendo mais frequentemente relatados ${ }^{(11-12)}$.

Neste estudo, os higromas predominaram no gênero masculino $(46,84 \%)$ e a mediana de idade foi de 55 anos, predominando em menores de 60 anos, semelhante ao observado em publicação realizada por Zanini et al, 2008 em nosso meio ${ }^{(11)}$.

Ao verificarmos os dados referentes à lateralidade, observamos que o lado esquerdo foi mais acometido em relação ao lado direito, todavia em números absolutos a bilateralidade foi mais frequente, conforme demostrado na tabela 4, o que contradiz os dados apresentados por Maher et al, 2013 ${ }^{(13)}$. Em relação às causas dos Higromas, não temos esta informação visto este estudo ser retrospectivo.

No que tange a abordagem cirúrgica, Ishibashi et al, 1994 ${ }^{(12)}$ demonstraram de forma consonante ao nosso trabalho que a trepanação foi o procedimento mais realizado tanto nos pacientes submetidos a uma intervenção cirúrgica quanto naqueles que necessitaram de reabordagem devido a recidiva, corroborando com os dados apresentados no presente estudo ${ }^{(12)}$.

\section{Conclusões}

As coleções subdurais crônicas não infecciosas representam grande parcela dos pacientes submetidos à intervenção neurocirúrgica, principalmente acima de 60 anos de idade.

A partir dos dados obtidos neste estudo é possível traçar o perfil epidemiológico dos pacientes com coleções subdurais crônicas não infecciosas atendidas em nosso serviço. Notamos que em sua maioria, os HSDCr representam o grande contingente das coleções subdurais submetidas a tratamento neurocirúrgico. É mais prevalente no gênero masculino, em pacientes de idade superior a 60 anos. Já em relação ao tratamento proposto, a trepanação foi o tratamento cirúrgico de escolha.

\section{Referências}

1. Baechli H, Nordmann A, Bucher HC, Gratzl O. Demographics and prevalent risk factors of chronic subdural haematoma: results of a large single-center cohort study. Neurosurg Rev. 2004; 27(4): 263-6.

2. Balser D, Rodgers SD, Johnson B, Shi C, Tabak E, Samadani U. Evolving management of symptomatic chronic subdural hematoma: experience of a single institution and review of the literature. Neurol Res. 2013; 35(3):233-42.

3. Lee KS, Bae WK, Bae HG, Yun IG. The fate of traumatic subdural hygroma in serial computed tomographic scans. J Korean Med Sci. 2000; 15(5):560-8.

4. Sousa EB, Brandão LF, Tavares CB, Borges IB, Freire Neto NG, Kessler IM. Epidemiological characteristics of 778 patients who underwent surgical drainage of chronic subdural hematomas in Brasília, Brazil. BMC Surg. $2013 ; 1 ; 13: 5$.

5. Ramachandran R, Hegde T. Chronic subdural hematomas causes of morbidity and mortality. Surg Neurol. 2007; 67(4):36772.

6. Nayil K, Ramzan A, Sajad A, Zahoor S, Wani A, Nizami F, et al. Subdural hematomas: an analysis of 1181 Kashmiri patients. World Neurosurg. 2012; 77(1):103-10.

7. Yang W, Huang J. Chronic subdural hematoma epidemiology and natural history. Neurosurg Clin N Am. 2017; 28(2):205-10.

8. Hsieh CT, Su IC, Hsu SK, Huang CT, Lian FJ, Chang CJ. Chronic subdural hematoma: differences between unilateral and bilateral occurrence. J Clin Neurosci. 2016;34:252-8.

9. Abecassis IJ, Kim LJ. Craniotomy for Treatment of Chronic Subdural Hematoma. Neurosurg Clin N Am. 2017; 28(2):229-37.

10. Gernsback J, Kolcun JPG, Jagid J. To drain or two drains: recurrences in chronic subdural hematomas. World Neurosurg. 2016; 95: 447-50.

11. Zanini MA, de Lima Resende LA, de Souza Faleiros AT, Gabarra RC. Traumatic subdural hygromas: proposed pathogenesis based classification. J Trauma. 2008; 64(3):705-713.

12. Ishibashi A, Yokokura Y, Miyagi J. Clinical analysis of nineteen patients with traumatic subdural hygromas. Kurume Med J. 1994; 41(2):81-5.

13. Maher CO, Garton HJ, Al-Holou WN, Trobe JD, Muraszko KM, Jackson EM. Management of subdural hygromas associated with arachnoid cysts. J Neurosurg Pediatr. 2013; 12(5):434-43.

Trabalho recebido: 09/04/2018

Trabalho aprovado: 16/07/2018 